\title{
Instrumental responses become associated with reinforcers that differ in one feature
}

\author{
ROBERT A. RESCORLA \\ University of Pennsylvania, Philadelphia, Pennsylvania
}

\begin{abstract}
In three experiments, thirsty rats were trained to make several instrumental responses whose outcomes differed in which of two relatively inconsequential flavor features they contained. In Experiment 1, one of the features was subsequently devalued by pairing it with lithium chloride; in Experiment 2, it was enhanced in value by pairing it with sucrose. In both experiments, differences in the value of the features resulted in parallel differences in the likelihood of the responses during a subsequent extinction test. In Experiment 3, the animals chose between these responses in the presence of discriminative stimuli that had signaled the occurrence of these different features following another response. The stimuli selectively augmented the likelihood of the response with which they shared training by the same-flavored consequence. These results indicate that rats can separately encode features that differ along one dimension, both in the association between an instrumental response and its outcome, and in the association between a discriminative stimulus and that outcome.
\end{abstract}

There is now clear evidence that associations form between an instrumental response $(R)$ and its reinforcing outcome $(\mathrm{O})$. In our laboratory, we have used two techniques to demonstrate and analyze such associations. One technique employs the devaluation of the outcome following instrumental training. Such a devaluation routinely decreases the likelihood of responses that had previously earned that outcome, while leaving responses that had earned other, nondevalued, outcomes unaffected (e.g., see Colwill \& Rescorla, 1985, 1986, 1990). This result implies the presence of highly specific $\mathrm{R}-\mathrm{O}$ associations. The second technique involves the transfer of control of a stimulus from one response to another, based on their shared outcomes. For instance, Colwill and Rescorla (1988a) conducted discrimination training in which a stimulus signaled that a response would be reinforced with a particular outcome. They found that that stimulus was able to augment the likelihood of another response with which it had never been trained, if the new response had earned the same outcome. However, the stimulus had little effect on a new response that had earned a different outcome. This result also implies the presence of highly specific $\mathrm{R}-\mathrm{O}$ associations. The conclusion that follows from both of these techniques is that the organism represents in some detail the outcome that reinforces a response.

This research was supported by National Science Foundation Grants BNS 83-08176 and BNS 88-03514 to the University of Pennsylvania. I would like to thank Ruth Colwill for many helpful discussions, and Michelle Klein for her assistance with data collection. Requests for reprints should be addressed to the author at the Department of Psychology, University of Pennsylvania, 3815 Walnut Street, Philadelphia, PA 19104.
The purpose of the present experiments was to explore further the richness of these outcome representations. The outcomes used by Colwill and Rescorla (1985) were a standard food pellet and liquid sucrose. Although both of these outcomes are attractive for food-deprived rats, they differ along many stimulus dimensions. In the present experiments, an attempt was made to reduce the differences between the stimuli to a single feature. Moreover, a differentiating feature that was largely irrelevant to the motivational state of the animal, and hence to the attractive value of that outcome, was selected. For this purpose, water-deprived animals were allowed to make two responses, both of which earned water; however, the water following one response was slightly bitter and that following the other response was slightly sour. The degree to which the sour and bitter components of the outcomes were differentially associated with the different responses was assessed using the two techniques described above. The issue is whether the outcome of instrumental behavior is represented with sufficient richness to support differential behavior based on a single relatively inconsequential feature.

\section{EXPERIMENT 1}

In this experiment, a devaluation technique was used to examine the specificity of outcome encoding. The procedure was modeled directly on that used by Colwill and Rescorla (1985). Each animal received training with two responses (leverpress and chain pull), one leading to water containing quinine and one leading to water containing weak hydrochloric acid $(\mathrm{HCl})$. The animals were then made ill following separate free delivery of water containing either $\mathrm{HCl}$ or quinine, and were tested for their preference between the two responses. As in earlier work, 
specific $\mathrm{R}-\mathrm{O}$ associations would be indicated by a bias against the response that had previously produced the outcome that was then devalued.

\section{Method}

Subjects. The subjects were 16 male Sprague-Dawley rats about 90 days old. They were housed in individual cages and were maintained on a water-deprivation regimen that permitted them $20 \mathrm{~min}$ of access to water daily after each session. They had free access to food in their home cages.

Apparatus. The apparatus consisted of eight identical operant chambers measuring $22.9 \times 20.3 \times 20.3 \mathrm{~cm}$. The two end walls of each chamber were aluminum; the side walls and ceiling were clear Plexiglas. Each chamber had a recessed food magazine in the center of one end wall. A small metal cup measuring $1.25 \mathrm{~cm}$ in diameter and $1.5 \mathrm{~cm}$ deep was sunk in the floor of each food magazine. To the left of the magazine was a lever and to the right was a chain suspended from a relay mounted on the roof of the chamber. Access to these manipulanda could be blocked by covering the lever with a metal shield and by retracting the chain through a hole in the ceiling. The floor of the chamber was composed of $0.48-\mathrm{cm}$ stainless steel rods, spaced $1.9 \mathrm{~cm}$ apart. Each chamber was enclosed in a sound- and light-resistant shell. A speaker that permitted the presentation of a white noise measuring approximately $76 \mathrm{~dB}$ re $20 \mu \mathrm{N} / \mathrm{m}^{2}$ against a background level of $62 \mathrm{~dB}$ was mounted on the inside wall of the shell. The outside ceiling of the shell supported a solenoid-operated gravity feed valve that was connected with plastic tubing to the cup in the food magazine. This system permitted the presentation of $0.3 \mathrm{ml}$ of distilled water alone, water containing sufficient quinine to make a $0.0001-\mathrm{M}$ solution, or water containing sufficient $\mathrm{HCl}$ to yield a $0.005-\mathrm{M}$ solution. These concentrations were selected because they are similar to those used in previous research on the learning of inconsequential reinforcers of Pavlovian events (e.g., Rescorla \& Cunningham, 1978).

Experimental events were controlled and recorded automatically by relays and microprocessors located in an adjoining room.

Procedure. On the initial day of the experiment, the animals received 20 free deliveries of $0.3 \mathrm{ml}$ of water, delivered on a variable-time (VT) 1-min schedule. During this session, neither the lever nor the chain was present. On each of the next 2 days, one of the manipulanda was inserted into the chamber and the animal was allowed to earn 25 water deliveries on a continuous reinforcement schedule. The lever was present during the first of these 2 days and the chain was present during the second.

Beginning with the next day, the animals were run for two 20-min sessions each day, separated by approximately $1 \mathrm{~h}$. In each session, one manipulandum was present and liquid could be earned on a variable-interval (VI) schedule. On Day 1 , each response produced a water outcome on a VI 30 -sec schedule. On the following 5 days, each response earned water on a VI 60 -sec schedule. Water continued to be earned on the same VI 60 -sec schedule on each of the next 4 days, but for one response the water contained $\mathrm{HCl}$ and for the other it contained quinine.

On each of the next 6 days, the manipulanda were removed. The animals received three 2-day cycles that were intended to devalue either $\mathrm{HCl}$ or quinine while leaving the other outcome unchanged. In the first session of each cycle, the animals received free deliveries of either $\mathrm{HCl}$ or quinine and the session terminated with the administration of a $0.5 \%$ body weight injection of $0.6 \mathrm{M}$ lithium chloride ( $\mathrm{LiCl}$ ). Initially, 200.3 -ml deliveries of solution were given on a VT schedule in a 20-min session; but in order to avoid overflowing the magazines, deliveries ceased in the second and third cycles as each animal came to reject the substance. The outer shells of the chambers were left open during those cycles to permit direct monitoring of the animal's contact with the fluids. In the second session of each cycle, the animals received 20 deliveries of the alternative flavor without an injection at the end of the session.
On the day following the last devaluation cycle, each animal was given a choice between leverpressing and chain pulling. Both manipulanda were continuously present during a 10 -min session, but responding had no programmed consequence for the animal.

\section{Results and Discussion}

Initial response training proceeded without incident. On the final day of VI 60-sec training with the water outcome, the mean response rate, averaged over both manipulanda, was 9.7 responses per minute. The substitution of $\mathrm{HCl}$ and quinine as the outcomes produced no detectable change in response rate. The mean response rate varied from 9.2 to 10.0 responses per minute over the 4 days of training using the differential outcomes. There were no reliable differences in the rates of the responses that earned $\mathrm{HCl}$ and quinine, nor did response rate differ reliably from the rate produced by the water outcome. This suggests that the present $\mathrm{HCl}$ and quinine components were indeed relatively unimportant as components of the reinforcer. Although some evidence suggests that concentrations of $\mathrm{HCl}$ and quinine such as those used in the present experiment may have a slight negative impact on consumption (Rescorla \& Cunningham, 1978), they apparently differ little in consequence from the instrumental reinforcing power of plain water.

Devaluation with the administration of $\mathrm{LiCl}$ was quite rapid and highly specific. On the third cycle of this training, all animals continued to consume all of the nonpoisoned substance, but all showed complete rejection of the poisoned substance, after an initial lick.

The data of primary interest are from the test session, during which both manipulanda were available. Figure 1 shows the results of that session, plotted separately for the devalued and nondevalued reinforcers. Because responding did not differ as a function of response or outcome identity, the results have been collapsed across those variables. It is clear from the figure that the response that

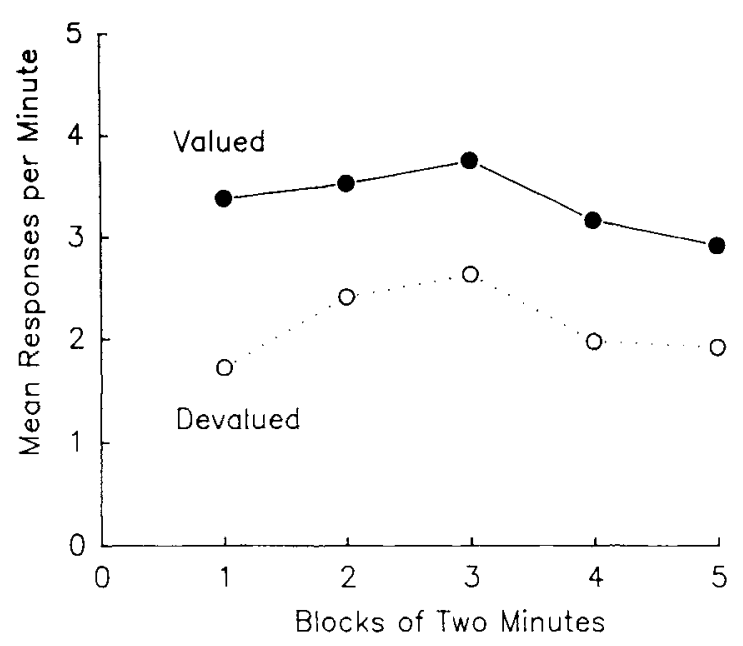

Figure 1. Responding during the test session of Experiment 1. Responding is shown separately for behaviors when the taste contained in its water outcome had been devalued by pairing it with LiCl or nondevalued. 
had previously earned the now-devalued outcome was depressed relative to the other response. There was little change in this pattern over the course of the brief test session. During the test session, $66 \%$ of the responses were to the manipulandum whose outcome had not been devalued. Largely because of substantial individual variation in the initial preference for the responses, the simple difference in response rates during the test session fell short of reliability [Wilcoxon $T(16)=34, .05<$ $p<.10$ ]. However, a measure that takes into account initial biases (i.e., the shift in preference for the nondevalued response as a function of devaluation) was highly reliable $[T(16)=20, p<.01]$. During the final training session, the mean percentage of responses that occurred for the to-be-devalued outcome was $50 \%$; after devaluation, that percentage was $34 \%$. This result suggests that the $\mathrm{HCl}$ and quinine components of the outcome were indeed encoded, despite their relative lack of effect at the time of their introduction.

The overall levels of responding observed during the test and the magnitude of the difference between the responses to the devalued and nondevalued manipulanda were both smaller in this experiment than in that reported by Colwill and Rescorla (1985). This is particularly clear for the response whose outcome was not devalued. One possibility is that there was greater generalization in the present experiment, perhaps due to the fact that the differentiating tastes were relatively less salient components of the outcomes. One may think of different outcomes as having the form $A X$ and $B X$, in which $A$ and $B$ represent the distinctive features and $X$ represents the shared features. It seems plausible that when $A$ and $B$ are relatively inconsequential features, such as $\mathrm{HCl}$ and quinine, the more salient common element would permit greater generalization between the outcomes. Even when such generalization is not apparent during the devaluation treatment, it might influence anticipatory responding.

It is worth commenting that simply observing performance during instrumental training would not have drawn attention to the fact that the added $\mathrm{HCl}$ and quinine features were being learned. Because these features have relatively little value, their introduction during the course of training led to no observed change in performance. Yet, on the basis of the devaluation manipulation, it is clear that they had in fact been encoded. This observation is similar to one made by Colwill and Rescorla (1988b) and suggests that, in some cases, manipulation of the value of an outcome after training has taken place can give a more complete picture of the learning than does simply looking at responding itself.

\section{EXPERIMENT 2}

In Experiment 1, the encoding of $\mathrm{HCl}$ and quinine was demonstrated by selective depression of a response when its outcome was devalued. In Experiment 2, an attempt was made to confirm this conclusion using a procedure that selectively enhances the value of one outcome rela- tive to the other. Because $\mathrm{HCl}$ and quinine are initially relatively innocuous, it may be possible to use a learning procedure to augment, rather than depress, their value. Consequently, in this experiment, different responses earned water containing either $\mathrm{HCl}$ or quinine. One of the flavors was then presented freely in conjunction with a more attractive substance, sucrose; this operation might be expected to enhance the value of the paired substance (e.g., see Fanselow \& Birk, 1982). Finally, the animals were given a choice between the two responses. In this experiment, the encoding of $\mathrm{HCl}$ or quinine as a component of the outcome in the $\mathrm{R}-\mathrm{O}$ association would be exhibited by a preference for the response whose outcome was subsequently presented in a sucrose solution.

\section{Method}

Subjects and Apparatus. The subjects were 16 male SpragueDawley rats maintained as in Experiment 1. The apparatus was that of Experiment 1. The concentrations of $\mathrm{HCl}$ and quinine were the same as in Experiment 1; when used, the sucrose concentration was $8 \% \mathrm{w} / \mathrm{v}$.

Procedure. All animals were initially magazine-trained with 10 free presentations of $\mathrm{HCl}$ followed by 10 presentations of quinine. On the following day, each animal received continuous reinforcement with the lever; for half of the animals, the outcome was $\mathrm{HCl}$; for the other half it was quinine. The next day, all animals received identical training with the chain, but using the alternative reinforcer. On the following 6 days, all animais received two 20 -min sessions per day, spaced approximately $1 \mathrm{~h}$ apart. In each session, one manipulandum was present and responding was reinforced with the appropriate outcome on a VI 60-sec schedule.

On each of the next 3 days, the animals received two sessions, one of which contained 20 free deliveries of $\mathrm{HCl}$ and the other of which contained 20 free deliveries of quinine. For half of the animals, $\mathrm{HCl}$ was presented in solution with $8 \%$ sucrose and quinine was presented in distilled water; for the other half of the animals, quinine was presented with sucrose and $\mathrm{HCl}$ was given alone.

On each of the next 4 days, the animals received a single 20-min test session. During these sessions, both the lever and the chain were continuously available, but neither manipulandum produced any programmed consequence.

\section{Results and Discussion}

Training proceeded smoothly with both manipulanda and outcomes. By the final day of training, the mean number of responses, collapsed across all treatments, was 8.2 responses per minute. There were no reliable differences as a function of response or outcome identity. The pretraining with water used in Experiment 1 was apparently not needed to ensure stable responding for water containing $\mathrm{HCl}$ and quinine.

The data of primary interest, from the test sessions, are shown in Figure 2, which displays responding during each of the test sessions, separated according to whether or not the response had had its outcome presented jointly with sucrose. Although there was initially no differential responding on the first test day, it is clear that a difference emerged, as responding extinguished. Over the last two test sessions, there were reliably more responses to the manipulandum whose outcome had received enhancement by pairing it with sucrose $[T(16)=21, p<.02]$. 


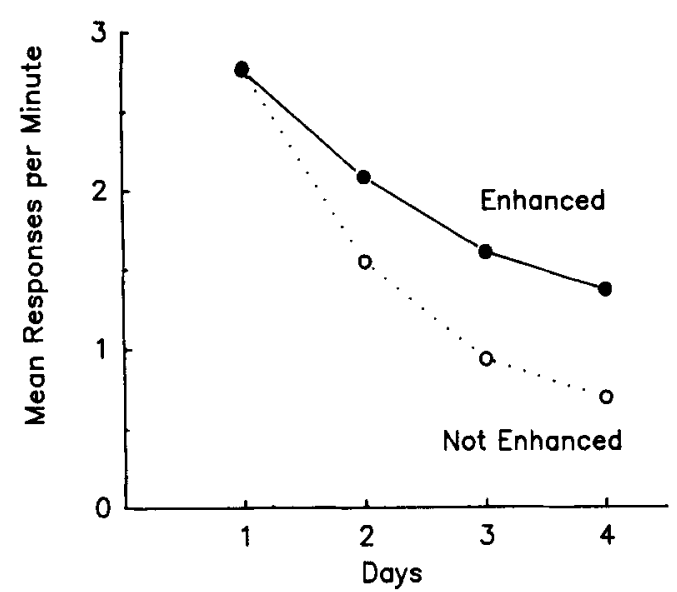

Figure 2. Responding over the four test sessions of Experiment 2. Responding is shown separately for behaviors when the taste contained in its water outcome had been enhanced in value by pairing it with sucrose or had not.

This confirms the conclusion of Experiment 1: There is differential encoding of $\mathrm{HCl}$ and quinine in rats as components of the outcomes associated with the different responses.

This experiment is unusual in that it was designed to measure instrumental responding after using a conditioning operation intended to enhance the attractiveness of a reinforcer. Previous revaluation experiments have used either a conditioning operation intended to reduce the attractiveness of a reinforcer (e.g., Colwill \& Rescorla, 1985 ) or a motivational manipulation intended to enhance its value (e.g., Dickinson \& Dawson, 1989; Krieckhaus $\&$ Wolf, 1968). It is not clear whether the present procedure will be generally useful for revaluing reinforcers, but it apparently is sufficiently powerful to change the value of features that are initially relatively neutral.

However, the results of this experiment should be interpreted with some caution. The procedure of delivering the different outcomes in small amounts in the operant chamber made it impossible to verify independently the success of the value-increase manipulation. As a result, it remains possible that some other consequent of the differential delivery of $\mathrm{HCl}$ and quinine with sucrose was responsible for the results. For instance, it might be the case that separate presentation of an outcome in itself has a suppressive effect on subsequent performance of the operant with which it was used, but that presentation with sucrose attenuates that effect. But whatever the detailed mechanism, it is clear that differential treatment of $\mathrm{HCl}$ and quinine had a differential effect on their responses, from which it may be concluded that the responses became associated with those different outcomes.

\section{EXPERIMENT 3}

In the previous two experiments, variations on the devaluation experiments of Colwill and Rescorla (1985) were used to identify encoding of $\mathrm{HCl}$ and quinine. In
Experiment 3, a variation on the transfer result described by Colwill and Rescorla (1988a) was used for the same purpose. As in the Colwill and Rescorla (1988a) experiment, two different stimuli signaled when a common response would yield reinforcement; but those stimuli uniquely specified which of two outcomes would follow that response. Then two other target responses were trained, one with each of those outcomes. Finally, the stimuli were presented while the animals had a choice between the target responses. Selective augmentation of the response with which a discriminative stimulus shared an outcome was taken as evidence for encoding of the details of the outcomes. The present experiment differed from that of Colwill and Rescorla (1988a) primarily in having the different outcomes specified in terms of $\mathrm{HCl}$ and quinine.

\section{Method}

Subjects and Apparatus. The subjects were 16 male SpragueDawley rats maintained as in Experiments 1 and 2. The apparatus was that of the previous experiments. It permitted the presentation of two stimuli: a 76-dB white noise $(N)$ and an illumination of a 6-W houselight ( $\mathrm{L})$ in an otherwise dark chamber. In addition, a nosepoke manipulandum could be made available by removing a cover on a $2-\mathrm{cm}$ circular hole located directly above the food magazine. Behind this hole was a plate that operated a microswitch when it was displaced.

Procedure. The animals received magazine training as in Experiment 2 . Then they received initial training with each of three responses-the lever, the chain, and the nosepoke manipulandum. On Day 1, each animal received continuous reinforcement training with the lever; half of the animals had the opportunity to earn 25 deliveries of $\mathrm{HCl}$ and half could earn 25 deliveries of quinine. On the following day, each animal received similar training with the chain, but with the alternative reinforcer. On Days 3 and 4, all animals received training with the nosepoke manipulandum, earning $\mathrm{HCl}$ on one day and quinine on the other. On the following 2 days, the animals received two 20-min sessions per day, spaced about $1 \mathrm{~h}$ apart. During each session, the nosepoke manipulandum was available and responding produced a fluid outcome on a VI 60-sec schedule; for one session of each day, the outcome was $\mathrm{HCl}$; for the other session it was quinine.

Discrimination training with $\mathrm{N}$ and $\mathrm{L}$ took place over the next 8 days. On each day, the animals received two sessions, spaced about $1 \mathrm{~h}$ apart, each containing $1630-\mathrm{sec}$ stimulus presentations. The noise stimulus was presented during one session of each day and the light stimulus was presented during the other session. During each stimulus presentation, a nosepoke earned a fluid outcome on a VI 30-sec schedule, with $\mathrm{L}$ signaling one outcome and $\mathrm{N}$ signaling the other. The intertrial intervals (ITIs) were 45,60 , and $90 \mathrm{sec}$ on the first 3 days of training, and $120 \mathrm{sec}$ thereafter.

On the next 3 days, the target responses (leverpress and chain pull) received VI 60-sec training, one with $\mathrm{HCl}$ and one with quinine. Assignment of outcomes to responses was carried out in a fashion orthogonal to the nosepoke training assignments. Each session was $20 \mathrm{~min}$ long and responding was reinforced on a VI 60-sec schedule. On the next day, all animals received a single $10-\mathrm{min}$ session during which both the lever and the chain were available but no reinforcers were delivered. The intention of this training, following the practice of Colwill and Rescorla (1988a), was to reduce the rate of the target responses in order to allow room for the stimuli to elevate responding in the subsequent test session.

On the next day, all animals received a single test session. During this session, both the lever and the chain were available continuously. Each stimulus was presented for eight 30 -sec periods in 
counterbalanced order, with a fixed 30 -sec interval between stimulus presentations. Responding had no programmed consequence. The question of interest was whether $\mathrm{N}$ and $\mathrm{L}$ would selectively elevate the response with which they shared the $\mathrm{HCl}$ or quinine outcome.

\section{Results and Discussion}

Initial response learning and discrimination training proceeded without incident. By the final day of discrimination training, both stimuli had acquired control over nosepoking. This control was expressed in terms of a discrimination ratio of the form $A /(A+B)$, where $A$ represents the response rate during the stimulus and $B$ represents the response rate in its absence. On Day 8 of discrimination training, the mean discrimination ratio was .88. No differences were observed as a function of stimulus or outcome identity.

Figure 3 shows responding during the test session, plotted in blocks of two presentations of each stimulus. The response rates in the absence of any stimulus (ITI) as well as during two types of stimuli (one sharing the same outcome with a response and one having a different outcome from the response) are shown separately. No differences were observed as a function of stimulus or response identity, so the data have been collapsed across those categories. Over the course of extinction testing, there was a general decline in responding in all periods. However, the same pattern of differences was present throughout testing. Responding was elevated relative to the ITI by both stimuli, but this elevation was greater when the stimulus shared the same outcome with the response. For the session as a whole, the stimulus reliably augmented both the same-outcome response $[T(16)=3$, $p<.01]$ and the different-outcome response $[T(16)=$ $20, p<.01]$. During the final two blocks of testing,

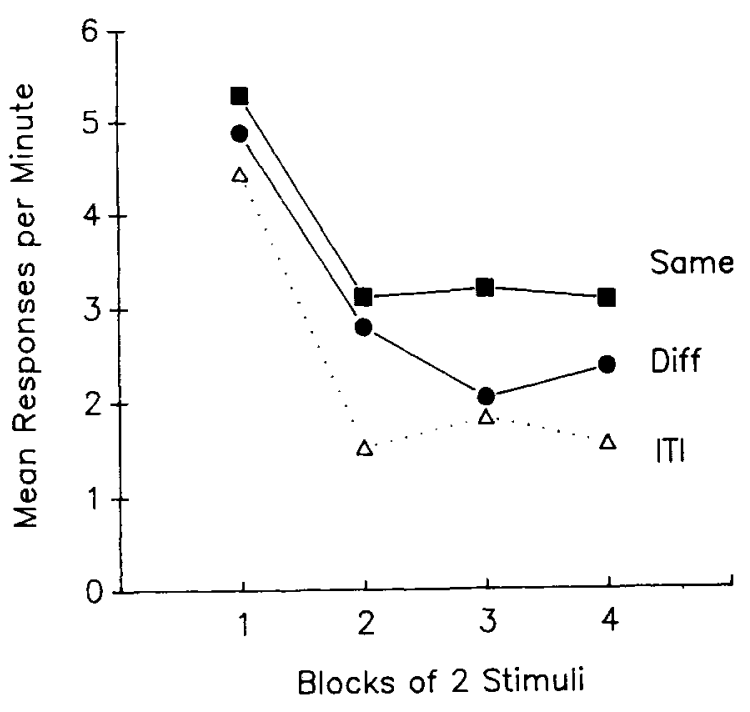

Figure 3. Responding during the test sessions of Experiment 3. Responding is shown separately in the absence of any stimulus (ITI) and in the presence of a stimulus that had predicted either the same or a different (Diff) flavor component as that which the response had produced in training. responding was reliably greater during the same stimulus than during the different stimulus $[T(16)=29$, $p<.05$ ].

These results are similar to those observed by Colwill and Rescorla (1988a) with sucrose and pellet outcomes, although the effects observed here appear to be generally weaker. The relatively smaller difference between the same and different conditions, as well as the ability of the different stimulus to augment responding somewhat relative to the ITI, implies less differentiation in the present study than in that of Colwill and Rescorla. However, the selective augmentation of responding on a manipulandum that shared the $\mathrm{HCl}$ or quinine component of the outcome with a stimulus implies that the rats had indeed encoded $\mathrm{HCl}$ and quinine as components of their association with the responses. The finding of a differential effect and the observation that the effect is apparently smaller than that observed in a previous study both agree with the conclusions of Experiment 1 . The potentially greater generalization between outcomes whose differentiating features are less salient is a natural interpretation.

It is also important to note that this selective augmentation additionally implies that the rats had encoded $\mathrm{HCl}$ and quinine as components of the outcomes associated with each stimulus. In order to show selective transfer of the sort observed here, in which responding depends on the stimulus and the response sharing an outcome, the subjects must have learned both the R-O and the S-O associations. Consequently, this experiment suggests a fine resolution on the part of the subjects with regard to both the outcomes they associated with their responses and those they associated with discriminative stimuli.

\section{GENERAL DISCUSSION}

These experiments provide two kinds of demonstration that animals encode the outcomes of instrumental responding in sufficient detail to permit distinctions among outcomes differing in a single, relatively inconsequential feature. First, when the differentiating features of the outcomes were revalued following instrumental training, the likelihood of the associated response was appropriately affected: the response likelihood was lower for a feature that had been devalued and higher for one that had been enhanced in value. Second, transfer of control of a stimulus from one instrumental act to another was partially mediated by a shared feature of the outcome. Such mediation could occur only if the representation of the outcome associated with both the stimulus and the response contained information about the feature.

The results of the revaluation experiments agree with earlier reports for relatively inconsequential features. For instance, the classic paper of Tolman and Gleitman (1949) describes how presenting shock in a goalbox that had previously been located at the end of one arm of a T-maze produced a differential reduction in the response that led to that goalbox. In effect, the stimulus properties of the goalbox were inconsequential features of the goal to which 
the instrumental response led; yet they were apparently encoded. Similarly, St. Claire-Smith and MacLaren (1983) paired shock or food with auditory and visual stimuli that had been consequent upon instrumental responding. They found those operations to reduce and augment, respectively, the likelihood of that response. The present Experiments 1 and 2 confirm those results with a somewhat more comprehensive within-subject design.

The transfer results of Experiment 3 suggest that the outcome features mediating control over a response by a stimulus need not themselves be highly valued by the animal. This conclusion is in agreement with the results reported by Colwill and Rescorla (1990). In that study, the transfer of a stimulus to a new response based on sharing either sucrose or pellet outcomes was investigated. The finding of interest is that the transfer continued even after devaluation of the outcome that the stimulus and response shared. As long as the target response was associated with some outcome that continued to have value, it was not necessary that the outcome mediating transfer continue to be valued by the organism. In effect, making the feature of an outcome responsible for transfer inconsequential after learning in their experiment had the same result as having it be inconsequential from the outset in the present Experiment 3.

Historically, theoretical views of the instrumental reinforcer have adopted a variety of positions on the richness of its encoding. Classical S-R reinforcement theories see the reinforcer as an event that promotes an association between a stimulus and a response without itself ever becoming encoded. In such views, the important property of the reinforcer is its affective value, with positive outcomes promoting the association and (according to some) negative outcomes undermining that association. By contrast, other historically prominent theorists (e.g., Tolman, 1932) have emphasized the importance of the stimulus properties of the reinforcer. They view the reinforcer as a stimulus whose features enter into the association. Twoprocess theories, in which the instrumental reinforcer also serves as a Pavlovian unconditioned stimulus (US), have occupied a middle ground. Some (e.g., Rescorla \& Solomon, 1967) have pointed to the motivational contribution of the Pavlovian process, emphasizing the affective value of the US. Others (e.g., Trapold \& Overmier, 1972) have instead noted the importance of the stimulus properties of the reinforcer as mediators of specific responses. It now seems clear that the extreme S-R reinforcement position is untenable; clearly stimulus features of the reinforcer are encoded, partly in terms of R-O associations. The observations made here, that even single relatively innocuous features of the reinforcer are encoded, help both to reject the $S-R$ view and to encourage alternatives in which the associative contribution of a reinforcer is viewed predominantly in terms of its stimulus features.

The results observed here parallel those found with similar techniques for Pavlovian conditioning. For instance, Rescorla (1980), working with second-order autoshaping in pigeons, found considerable encoding of a relatively neutral feature of the reinforcing stimulus. This encoding was detected by showing that responding to a signal of the reinforcer was affected by manipulation of the value of the neutral feature. Indeed, in that preparation, neutral features seem to be encoded as well as more valued features. It is not clear whether the same will be the case for encoding features of instrumental reinforcers.

In any case, the present data encourage the view that many different features of an outcome are encoded both by stimuli and by responses that precede that outcome. Rats appear to develop quite a detailed representation of the outcome. Moreover, even features that are relatively inconsequential can play a role in governing the likelihood of responding.

\section{REFERENCES}

Colwill, R. M., Rescorla, R. A. (1985). Post-conditioning devaluation of a reinforcer affects instrumental responding. Journal of Experimental Psychology: Animal Behavior Processes, 11, 120-132.

Colwill, R. M., Rescorla, R. A. (1986). Associative structures in instrumental learning. In G. H. Bower (Ed.), The psychology of learming and motivation (Vol. 20, pp. 55-104). New York: Academic Press.

Colwill, R. M., Rescorla, R. A. (1988a). Associations between the discriminative stimulus and the reinforcer in instrumental learning. Joumal of Experimental Psychology: Animal Behavior Processes, 14, 155-164.

Colwill, R. M., Rescorla, R. A. (1988b). The role of responsereinforcer association increases throughout extended instrumental training. Animal Learning \& Behavior, 16, 105-111.

Colwill, R. M., \& ResCoRla, R. A. (1990). Effect of reinforcer devaluation on discriminative control of instrumental behavior. Journal of Experimental Psychology: Animal Behavior Processes, 16, $40-47$.

Dickinson, A., Dawson, G. R. (1989). Incentive leaming and the motivational control of instrumental performance. Quarterly Journal of Experimental Psychology, 41B, 99-112.

FANSELOW, M. S., BIRK, J. (1982). Flavor-flavor associations induce hedonic shifts in taste preference. Animal Learning \& Behavior, 10, 223-228.

KrieckHaus, E. E., Wolf, G. (1968). Acquisition of sodium by rats: Interaction of innate mechanisms and latent learning. Journal of Comparative \& Physiological Psychology, 65, 197-201.

Rescorla, R. A. (1980). Pavlovian second-order conditioning: Studies in associative learning. Hillsdale, $\mathrm{NJ}$ : Erlbaum.

Rescorla, R. A., CunNingham, C. L. (1978). Within-flavor associations. Jourmal of Experimental Psychology: Animal Behavior Processes, 4, 267-275.

Rescorla, R. A., Solomon, R. L. (1967). Two-process learning theory: Relationships between Pavlovian conditioning and instrumental learning. Psychological Review, 74, 151-172.

St. Claire-Smith, R., Maclaren, D. (1983). Response preconditioning effects. Joumal of Experimental Psychology: Animal Behavior Processes, 9, 41-48.

Tolman, E. C. (1932). Purposive behavior in animals and men. New York: Century.

Tolman, E. C., \& Gleitman, H. (1949). Studies in learning and motivation: I. Equal reinforcements in both end-boxes, followed by shock in one end-box. Joumal of Experimental Psychology, 39, 810-819.

Trapold, M. A., \& Overmier, J. B. (1972). The second leaming process in instrumenntal learning. In A. H. Black \& W. F. Prokasy (Eds.), Classical conditioning: Vol. 2. Current research and theory (pp. 427452). New York: Appleton-Century-Crofts.

(Manuscript received September 12, 1989; revision accepted for publication January 3, 1990.) 